\title{
Evidence for the Involvement of Lfc and Tctex-1 in Axon Formation
}

\author{
Cecilia Conde, ${ }^{1}$ Cristina Arias, ${ }^{1}$ Maria Robin, ${ }^{1}$ Aiqun Li, ${ }^{2}$ Masaki Saito, ${ }^{2}$ Jen-Zen Chuang, ${ }^{2}$ Angus C. Nairn, ${ }^{4}$ \\ Ching-Hwa Sung, ${ }^{2,3}$ and Alfredo Cáceres ${ }^{1}$ \\ ${ }^{1}$ Instituto de Investigación Médica Mercedes y Martín Ferreyra-Consejo Nacional de Investigaciones Científicas y Técnicas, 5016 Cordoba, Argentina, \\ Departments of ${ }^{2}$ Ophthalmology and ${ }^{3}$ Cell and Developmental Biology, Weil Medical College of Cornell University, New York, New York 10012, and \\ ${ }^{4}$ Department of Psychiatry, Yale University, New Haven, Connecticut 06511
}

RhoA and Rac play key and opposite roles during neuronal polarization. We now show that $\mathrm{Lfc}$, a guanosine nucleotide exchange factor (GEF), localizes to the Golgi apparatus and growth cones of developing neurons and negatively regulates neurite sprouting and axon formation through a Rho signaling pathway. Tctex-1, a dynein light chain implicated in axon outgrowth by modulating actin dynamics and Rac activity, colocalizes and physically interacts with Lfc, thus inhibiting its GEF activity, decreasing Rho-GTP levels, and functionally antagonizing Lfc during neurite formation.

\section{Introduction}

Activation of a signaling pathway involving a receptor tyrosine kinase (Sosa et al., 2006), phosphatidylinositol 3-kinase (PI3K) (Shi et al., 2003), Par polarity complex (Shi et al., 2003), and small Rho GTPases (Schwamborn and Puschel, 2004; Chuang et al., 2005; Nishimura et al., 2005) is crucial for axon formation. This facilitatory pathway is reinforced by the action of a positive feedback loop involving guanosine nucleotide exchange factors (GEFs) for Rac (Arimura and Kaibuchi, 2007; Barnes and Polleux, 2009; Conde and Cáceres, 2009). Overexpression of either of the GEFs, TIAM1 or STEF, induces multiple axon formation, whereas their depletion prevents axon outgrowth (Kunda et al., 2001; Nishimura et al., 2005). TIAM1 and STEF directly interact with PAR3 forming a complex with PAR6-atypical protein kinase C and GTP-bound Cdc42 that leads to Rac activation (Nishimura et al., 2005). Since GTP-bound Rac also activates PI3K (Tolias et al., 1995; Keely et al., 1997; Chan et al., 2002), a positive feedback loop is thus generated to drive the continued activation of PI3K.

In contrast, a negative feedback loop involving RhoA may serve to prevent formation of supernumerary axons during polarization. Overexpression of RhoA prevents axon formation, whereas expression of dominant-negative RhoA or inhibition of its downstream effector Rho kinase (RhoK) results in multiple axon formation (Da Silva et al., 2003; Chuang et al., 2005; Sanchez et al., 2008). Moreover, the RhoA and Rac pathways appear to have a tug-of-war relationship. For example, RhoK phosphorylates and inhibits TIAM1, STEF, and PAR3; disrupts the polarity complex; and prevents Rac activation (Nakayama et

Received Nov. 2, 2009; revised Jan. 5, 2010; accepted March 29, 2010.

This work was supported by grants from FONCyT, CONICET, Agencia Cordoba Ciencia (to A.C. and C.C.), Research to Prevent Blindness, and the Starr Foundation (to C.-H.S.) and by National Institute of Drug Abuse Grant DA10044 (to A.C.N.) and National Institutes of Health-National Eye Institute Grant R01 EY11307. C.A. is a PhD student from the School of Biology, National University Cordoba, Cordoba, Argentina.

Correspondence should be addressed to Alfredo Cáceres, INIMEC-CONICET, Av. Friuli 2434, 5016 Cordoba, Argentina.E-mail: acaceres@immf.uncor.edu.

DOI:10.1523/JNEUROSCI.5420-09.2010

Copyright $\odot 2010$ the authors $\quad 0270-6474 / 10 / 306793-08 \$ 15.00 / 0$ al., 2008). Therefore, a mechanism may exist to inhibit the RhoRhoK pathway to maintain an active PAR-TIAM1/STEF-Cdc42Rac module for axon specification (Conde and Cáceres, 2009). At present, the mechanisms underlying the regulation of Rho-RhoK during neuronal polarization are primarily unknown.

We have previously shown that a dynein light chain, Tctex-1, is required for axon formation by modulating a Rho-GTPasedependent pathway (Chuang et al., 2005). Neurons overexpressing Tctex-1 had elevated Rac activity, and functional analysis showed that dominant-negative Rac or constitutively active RhoA counteracted Tctex-1-mediated axon outgrowth. Since Tctex-1 has no GEF activity, it is likely that modulation of RhoGTPase activity occurs indirectly. We now show that Tctex-1 physically interacts with the Rho-GEF, Lfc, and functionally antagonizes its activity during neurite formation.

\section{Materials and Methods}

DNA constructs and two-hybrid screening. The Lfc short hairpin (sh)-RNA plasmids were constructed in pBS/U6 vector. 5'-GGGCTGCGGTTGCTTCTGTAA-3' (sh1-Lfc), 5'-GGGATGCTGGAAGAGTTGCAG-3' (sh2Lfc), and 5'-GGGTATTCGGTTGACCCTGTG-3' (scrambled for sh1, SC-sh) were used as targeting sequences following the procedures described by Chuang et al. (2005). The DNA fragments containing U6-Lfc-sh1, U6Lfc-sh2, or U6-SC-sh were inserted into pCAG-HcRed vector in which the HcRed-cDNA is under the control of chick actin-minimal (CAG) promoter; the resulting plasmids were referred to as sh1-Lfc-HcRed, sh2-Lfc-HcRed, and SC-sh-HcRed, respectively. The construction of plasmids encoding Flag-Tctex-1, Flag-rp3, GST-Tctex-1, Lfc-GFP, and Lfc-GST has been described previously (Chuang et al., 2005; Ryan et al., 2005); vsvg-Lfc was a generous gift from Dr. K. Matter (University College London, London, UK) (Aijaz et al., 2005). Two-hybrid screening was performed essentially as described by Tai et al. (1999) using Lfc as prey and Tctex-1 as bait.

Culture, transfection, immunofluorescence, and morphometry. Neuronal cultures from embryonic rat hippocampi were prepared as described previously (Kunda et al., 2001; Rosso et al., 2004; Chuang et al., 2005). Transient transfection of cultured neurons was performed with Lipofectamine 2000 as described previously (Chuang et al., 2005), and the constructs were used at concentrations ranging from 2 to $4 \mu \mathrm{g} / \mathrm{ml}$. Neurons were fixed with $4 \%$ paraformaldehyde in $4 \%$ sucrose-containing 
PBS and permeabilized in $0.2 \%$ Triton X-100 in PBS for 5 min before antibody incubation; for some experiments, cultures were extracted with detergents under microtubule-stabilizing conditions before fixation to obtain cytoskeletal preparations (Kunda et al., 2001). The following primary antibodies were used in this study: a monoclonal antibody $(\mathrm{mAb})$ against tau protein (clone Tau-1; a generous gift from Dr. L. I. Binder, Northwestern University, Chicago, IL), diluted 1:100; a mAb against tyrosinated $\alpha$-tubulin, diluted 1:1000 (Sigma); a mAb and a polyclonal antibody against Tctex-1 (Chuang et al., 2005), diluted 1:800; an affinity-purified rabbit polyclonal antibody raised against $\mathrm{Lfc}$ (Santa Cruz Biotechnology), diluted 1:50 or 1:100; a chicken polyclonal antibody against rat $L f c$, diluted 1:10 or 1:20; a rabbit polyclonal antibody or a mouse mAb against Flag (Sigma), diluted 1:500; and a rabbit polyclonal antibody against glutathione $S$-transferase (GST) (Santa Cruz Biotechnology). For some experiments, rhodamine-phalloidin (1:1000; Invitrogen) was used to stain filamentous actin (F-actin). Cells were visualized using either a spectral (Olympus Fluoview 1000) inverted confocal microscope or a total internal reflection fluorescence (TIRF) microscope (Nikon TE 2000) as described previously (Bisbal et al., 2008). In addition, the relative intensities of LfC and HcRed were evaluated using quantitative fluorescence techniques as described previously (Chuang et al., 2005). Cells were visualized with an inverted Zeiss microscope, and images ( 8 bites) were collected using a CCD camera (Orca 1000; Hamamatsu) and MetaMorph software (Molecular Devices). Fluorescence intensity measurements were performed within the cell body and neurites of identified neurons; with these data, the average fluorescence intensity expressed in pixels $(0=$ black $/$ $255=$ white) was calculated within the cell body and within the inner, middle, and distal third of identified neurites. Background levels were those detected in unlabeled cells. Images were processed using Adobe Photoshop. Neuronal shape parameters were evaluated as described previously (Chuang et al., 2005; Bisbal et al., 2008). Briefly, montages showing the complete neuronal arbor of transfected neurons were created from confocal images (maximal projections) acquired through a $60 \times, 1.4$ numerical aperture oil objective lens, and the number and length of neuritic processes were measured from a video screen using MetaMorph software. Differences among experimental groups were analyzed by one-way ANOVA and Tukey's post hoc test.

Rho-GTPase activity and $L f_{c}-G E F$ assays. Rho-GTPase activity was quantified by measuring the amounts of Rho precipitated in a pull-down reaction from cell lysates using GST-agarose containing the Rho-binding domain (Rhotekin-RBD) of a Rho effector coupled to agarose beads for GTP-bound Rho or an ELISA following the manufacturer's instructions (Cytoskeleton) (Chuang et al., 2005; Sosa et al., 2006; Sanchez et al., 2008). For Lfc guanine nucleotide exchange activity assay, fluorescence spectroscopic analysis of $\mathrm{N}$-methylanthraniloyl (mant)-GTP incorporation into bacterially purified Rho GTPases was performed according to the manufacturer's instructions (RhoGEF Exchange Assay; Cytoskeleton). Briefly, exchange reaction assay mixtures (150 $\mu$ l reaction volumes) containing an exchange reaction buffer (20 mM Tris, $\mathrm{pH} 7.5,50 \mathrm{~mm}$ $\mathrm{NaCl}, 10 \mathrm{~mm} \mathrm{MgCl} 2,50 \mu \mathrm{g} / \mathrm{ml}$ bovine serum albumin, $1 \mu \mathrm{M}$ mant-GTP) and $1 \mu \mathrm{M}$ RhoA-GTPase and 2-5 $\mu \mathrm{M}$ of each of the GST-fusion proteins (GEF-H1, Tctex-1, rp3) were used to analyze GEF activity. Reactions were measured with a Fluorometer ISS-K2 (multifrequency phase and modulation fluorometer), and the relative fluorescence $\left(\lambda_{\mathrm{ex}}=360 \mathrm{~nm}\right.$, $\lambda_{\text {em }}=460 \mathrm{~nm}$ ) was monitored at $20^{\circ} \mathrm{C}$ every $30 \mathrm{~s}$ for a total period of 30 min. Differences among experimental groups were analyzed by two-way ANOVA and Tukey's post hoc test.

Western blot and immunoprecipitation assays. Levels of $\mathrm{Lfc}$ and tubulin after RNA interference treatment in Chinese hamster ovary (CHO) cells or in cultured hippocampal pyramidal neurons were analyzed by Western blotting as described previously (Chuang et al., 2005). Immunoprecipitation and immunoblotting assays using extracts of transfected human embryonic kidney (HEK) cells or brain lysates were performed essentially as described previously (Chuang et al., 2005; Bisbal et al., 2009).

\section{Results}

\section{Subcellular distribution of Lfc during axon outgrowth}

The involvement of Lfc during spinogenesis has been examined in mature neuronal cultures (Ryan et al., 2005; Kang et al., 2009). Little is known, however, about the subcellular distribution or function of Lfc during the initial stages of process formation. Therefore, in initial experiments, we analyzed Lfc localization in young cultured hippocampal neurons, when they either display a symmetric array of short neurites (minor processes, stage 2 ) or 

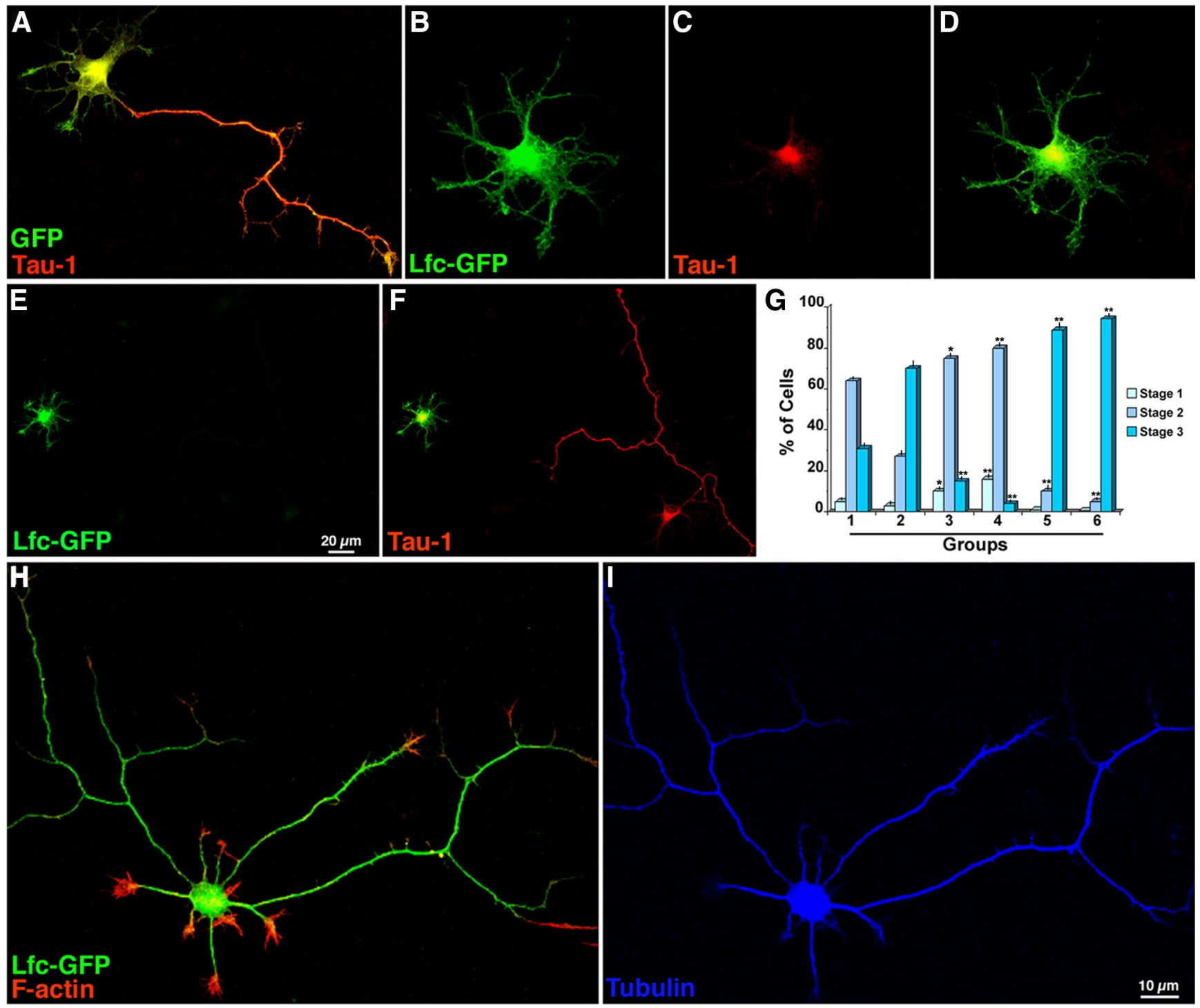

Figure 2. Lfc overexpression inhibits axon formation. $A$, Confocal image showing a cultured hippocampal neuron transfected with GFP (green) $2 \mathrm{~h}$ after plating, fixed $24 \mathrm{~h}$ later, and stained with a mAb against Tau (Tau-1; red). $\boldsymbol{B}-\boldsymbol{D}$, A similar experiment, but from a culture transfected with LfC-GFP. Note that the transfected cell is arrested at stage 2 of neuritic development; also note that Tau immunolabeling $(\boldsymbol{C})$ is restricted to the cell body. $\boldsymbol{E}, \boldsymbol{F}$, Another example of a neuron transfected with Lf(-GFP that has failed to develop an axon; in contrast, a nontransfected neuron present in the same field has extended a single long and branched Tau $+(\boldsymbol{F}$, red) axon. $\boldsymbol{G}$, Graph showing the percentage of cells at stages $1-3$ of neuritic development after transfection with either GFP or Lf-GFP; cultures were transfected $2 \mathrm{~h}$ after plating and fixed 24 or $30 \mathrm{~h}$ later. The different groups represent the following: 1, GFP (24 h); 2 , GFP ( $30 \mathrm{~h}) ; 3$, Lfc-GFP (24 h); 4, Lfc-GFP ( $30 \mathrm{~h}$ ); 5, Lfc-GFP plus Y27632 (24h); 6, Lfc-GFP plus Y27632 (30 h). Note that Lfc overexpression significantly ( ${ }^{*} p<0.05 ;{ }^{* *} p<0.001$ ) increases the number of cells at stages 1 and 2 while decreasing those at stage 3 of neuritic development. Also note that $Y 27632$ reverts the inhibitory effect of $L f c$ and significantly $\left({ }^{* *} p<0.001\right)$ increases the percentage of cells at stage 3 . $\boldsymbol{H}, \boldsymbol{I}$, Confocal images showing the morphology of an LfC-GFP-transfected neuron ( $\boldsymbol{H}$, green) treated with Y27632; the inhibitor ( $37 \mathrm{nmol} / \mathrm{ml})$ was added to the culture medium $2 \mathrm{~h}$ after transfection, fixed $24 \mathrm{~h}$ later, and labeled for tyr-tubulin (blue) and rhodamine-phalloidin (red). Note that the Lff-GFP-transfected neuron has extended several long axon-like neurites; a similar response was observed in control GFPtransfected neurons (data not shown).

have already developed an axon-like neurite (stage 3 ). Using two different antibodies (Fig. $1 \mathrm{~A}$ ), Lfc was found in stage 2 cells to be localized throughout the cytoplasm but to be prominent in the perinuclear region, presumably the Golgi apparatus (Fig. $1 B-D$ ), and also present at neuritic tips (Fig. $1 G, H$ ). During the transition through stages 2 and 3, Lfc immunofluorescence was found in the longest neurite (i.e., the prospective axon) that has a large growth cone (Bradke and Dotti, 1997; Kunda et al., 2001). Confocal microscopy revealed the presence of Lfc immunofluorescence associated with microtubules located in the central (C) and peripheral $(\mathrm{P})$ regions of the growth cone, as well as with actin ribs and filopodia in the $\mathrm{P}$ domain (Fig. $1 I-K$ ). After reaching stage 3, Lfc displayed a slightly polarized distribution, as it be- came enriched in the axonal shaft compared with the remaining minor processes (Fig. $1 L, M$ ).

\section{Lfc prevents axon formation}

Gain-of-function studies were subsequently performed to examine the role of Lfc in neurite sprouting and axon formation. Cultures were transfected with green fluorescent protein (GFP) or Lfc-GFP $2 \mathrm{~h}$ after plating and fixed 20-30 h later. GFPtransfected control neurons exhibited normal development through stages 2 and 3, at which time they displayed a single Tau1+ axon and minor processes (Fig. $2 A$ ). In contrast, almost all cells expressing Lfc-GFP were arrested at stage 2 (Fig. $2 B-F$ ). None of these cells were able to segregate Tau to a single neurite 
with most of the labeling found in the cell body (Fig. 2C). Morphometry revealed that although $>60 \%$ of the $30 \mathrm{~h}$ control neurons reached stage $3,<5 \%$ of Lfctransfected neurons reached this stage at this time point (Fig. 2G). A decrease in the number of primary neurites extended by stage 2 neurons and a significant increase in the number of stage 1 cells were also detected (Fig. 2G). Blocking RhoK activity with the inhibitor Y27632 reversed the effect of Lfc-GFP on axon formation (Fig. $2 G-I)$.

We then used a gene silencing approach to complement the experiments described above. For this purpose, we generated plasmids harboring both Lfc sh-RNA and HcRed cDNA. Immunoblotting (supplemental Fig. $1 A$, available at www.jneurosci.org as supplemental material) and immunostaining (supplemental Fig. $1 B-N$, available at www.jneurosci.org as supplemental material) showed that transfection with Lfc-sh-RNAs led to suppression of Lfc expression. Suppression of Lfc was accompanied by a significant reduction in endogenous Rho GTPase activity and Lfc-GFP-induced Rho activation (Fig. 3A). More importantly, the majority of neurons in which Lfc expression was reduced reached stage 3 of neuritic development, displaying much longer and branched axon-like neurites than control cells (Fig. 3B-L). In addition, neurons with 2 or 3 axon-like neurites (Tau-1+) were also detected. Finally, the stimulatory effect of Lfc suppression on axon formation was neutralized by coexpression of constitutively active RhoA (Fig. 3B).

To test whether or not Lfc prevents excessive axonal elongation and/or formation of supernumerary axons in neurons that have already acquired morphological polarity, cultures were transfected with the Lfc-sh-RNA plasmids $2 \mathrm{~d}$ after plating and examined $1 \mathrm{~d}$ later. Most $(86 \pm 4 \%)$ Lfc-suppressed neurons exhibited increased axonal length and branching and had two or more Tau-1+ axons (Fig. 4, Table 1). Together, our results support the existence of a Rho inhibitory tone that acts on minor neurites before and after polarization to prevent axon formation and suggest that this effect is mediated by the activity of a specific GEF, namely Lfc.

\section{Tctex-1 interacts and antagonizes Lfc activity during} axon development

Ectopic expression of Lfc in cultured hippocampal neurons not only induces Rho activation but also decreases Rac activity (Kang et al., 2009). We have previously shown that Tctex-1 is required for axon formation, an effect that involves modulation of the actin cytoskeleton through a Rho-GTPase-dependent pathway (Chuang et al., 2005). Recent reports suggest a potential interaction between Tctex-1 and Lfc (Gauthier-Fisher et al., 2009; Meiri et al., 2009). To test whether Tctex-1 and Lfc may functionally interact during neurite development, several assays were used. First, we used a yeast two-hybrid binary binding assay to show
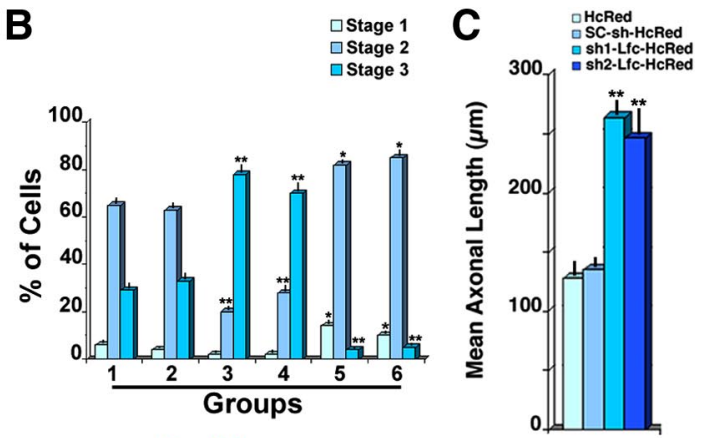

Merge
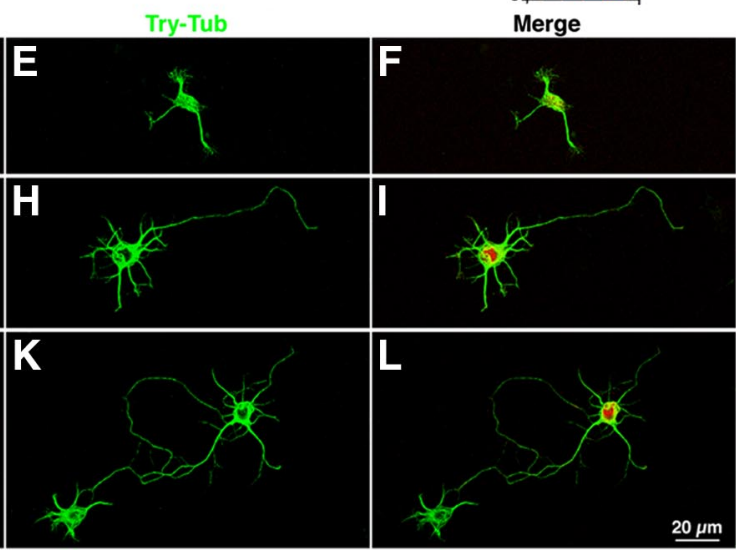

$0 \mu \mathrm{m}$

Figure 3. A, Graph showing levels of endogenous RhoA-GTP in SC-sh-HcRed-transfected (1) and sh1-LfC-HcRed-transfected (2) ippocampal pyramidal neurons. It also shows Rho-GTP levels after transfection with LfC-GFP (3) and Lfc-GFP plus sh1-Lfc-HcRed

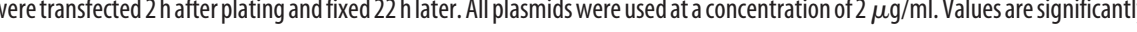
pal pyramidal neurons after transfection with SC-sh-HcRed and sh1-Lfc-HcRed. Neurons were transfected $2 \mathrm{~h}$ after plating, fixed at the end of the first day in vitro, and labeled with a mAb against tyrosinated $\alpha$-tubulin (green).

that Lfc specifically binds to Tctex-1 (Fig. 5A). Second, we used a coimmunoprecipitation assay to demonstrate that Tctex- 1 and Lfc interacted with each other inside cells. The anti-Flag antibody pulled down vsvg-Lfc along with Flag-Tctex-1 from extracts of HEK cells that coexpressed both of these proteins (Fig. 5B). Similar experiments that were performed with Flag-rp3, a homologous dynein light chain (Chuang et al., 2001; Vallee et al., 2004), failed to coimmunoprecipitate vsvg-Lfc (Fig. 5B). Finally, we showed that endogenous Tctex-1 coimmunoprecipitated with Lfc from embryonic mouse brain extracts (Fig. 5C).

Confocal microscopy revealed that Tctex-1 and Lfc colocalized within the $\mathrm{C}$ and $\mathrm{P}$ domains of the axonal growth cone, where they associated with microtubules and the actin cytoskeleton (Fig. 5D-F). Within the P domain, Tctex-1 immunolabeling predominated at the base of filopodial extensions, whereas Lfc was more enriched at the distal ends of filopodial protrusions or actin ribs. TIRFM also revealed similar colocalization with microtubules and F-actin-containing structures located in close proximity to the growth cone plasma membrane (Fig. $5 G-K$ ); Tctex-1, but not Lfc, was abundant at the transition zone between the $\mathrm{C}$ and $\mathrm{P}$ domains.

We then analyzed whether Tctex-1 was capable of regulating the GEF activity of Lfc. To this end, we expressed and purified several GST fusion proteins (Fig. $5 \mathrm{~L}$ ) and used a mant-fluorophore- 

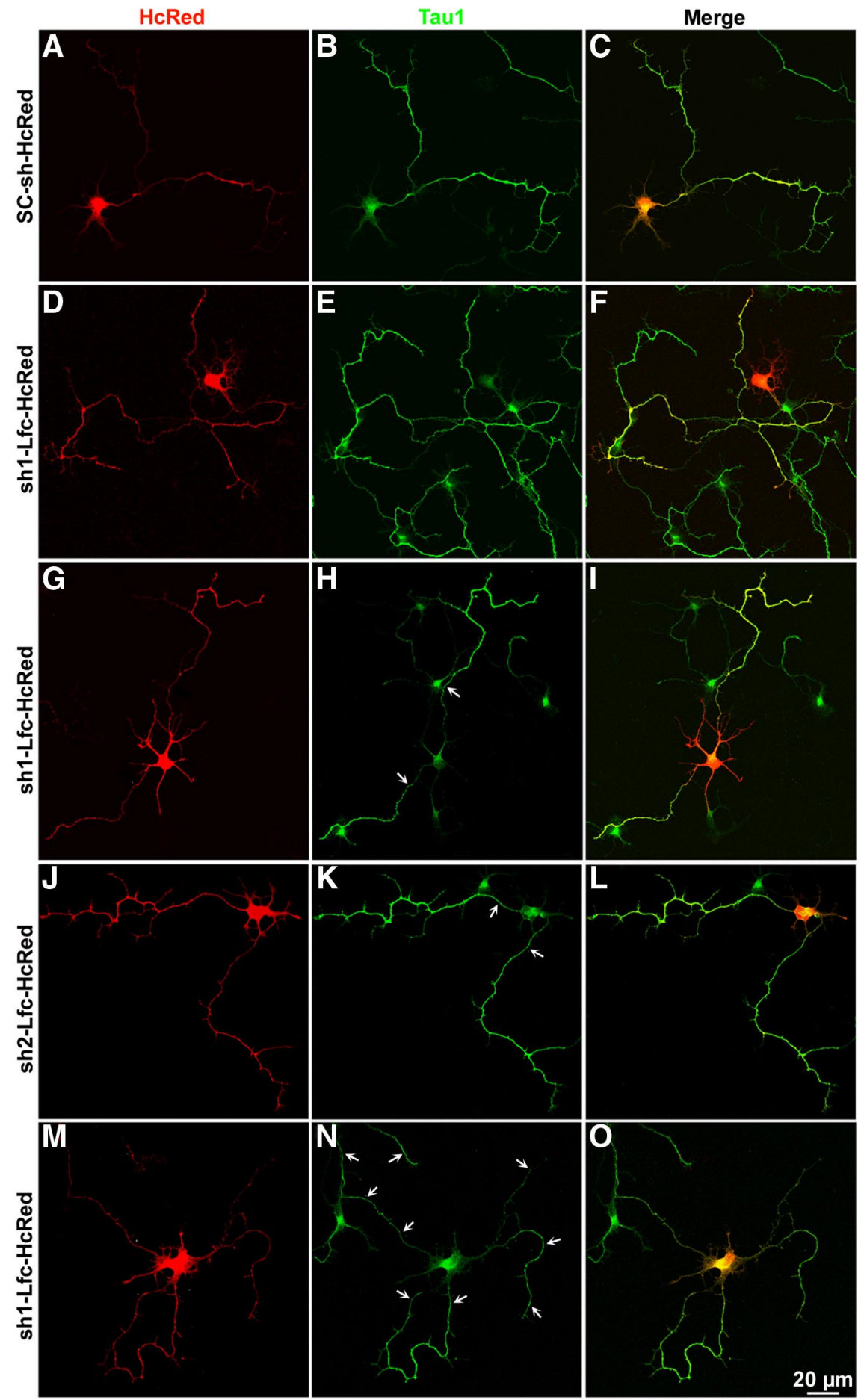

Figure 4. Lfc suppression enhances axon elongation and induces formation of supernumerary axons. $\boldsymbol{A}-\mathbf{0}$, Confocal images showing examples of neurons treated with control sh-RNA-HcRed (SC-sh-HcRed), sh1-Lfc-HcRed, or sh2-Lfc-HcRed. Cultures were transfected with the corresponding plasmids ( $4 \mu \mathrm{g}$ DNA each) $48 \mathrm{~h}$ after plating and fixed $1 \mathrm{~d}$ later. The cultures were counterstained with the mAb Tau-1 (green). Note that Lfc suppression increases axonal length, collateral branching, and the formation of supernumerary axons (arrows).

based GEF assay (see Materials and Methods) to test the effect of dynein light chains on Lfc activity. The results showed that Tctex-1, but not rp3, significantly inhibited Lfc activity in a dosedependent manner (Fig. $5 \mathrm{M}, \mathrm{N}$ ). This was consistent with pulldown assays or ELISAs, which revealed that Tctex-1 overexpression significantly reduced Lfc-induced RhoA activity in $\mathrm{CHO}$ cells (supplemental Fig. $2 A, B$, available at www.jneurosci.org as supplemental material), without affecting total RhoA levels. No effect on Lfcinduced RhoA activation was detected after coexpression of rp3 (supplemental Fig. $2 B$, available at www.jneurosci.org as supplemental material). Together, these biochemical and immunochemical data consistently suggest that Tctex-1 interacts with Lfc and inhibits its GEF activity, thereby decreasing Rho-GTP levels.

In the final set of experiments, we examined whether Tctex-1 overexpression could compensate for the inhibitory effect of Lfc on axon development. Neurons cotransfected with equal amounts of FlagTctex-1 and Lfc-GFP displayed a single axon with several minor neurites (Fig. 6A-C; supplemental Fig. 3, available at www.jneurosci.org as supplemental material), whereas those transfected with Tctex-1 and Lfc-GFP in a 2:1 ratio displayed multiple axon-like neurites (Fig. $6 D-I)$. In contrast, Flag-rp3 did not influence the inhibitory effect of Lfc on axon formation (supplemental Fig. 3, available at www.jneurosci.org as supplemental material).

\section{Discussion}

\section{$\mathrm{Lfc}$ is a negative regulator of} axon formation

Previous studies have shown that the RhoA-RhoK pathway exerts an inhibitory influence during the entire neuronal morphogenetic program (Govek et al., 2005; Sordella and Van Aelst, 2008; Tashiro and Yuste, 2008). In the particular case of axogenesis, activation of the RhoARhoK pathway suppresses axon outgrowth, whereas its suppression/inactivation enhances axonal formation (Bito et al., 2000; Borisoff et al., 2003; Da Silva et al., 2003; Chuang et al., 2005; Sanchez et al., 2008). It follows that tight spatial and temporal control of RhoA-RhoK activity should be a critical feature of neuronal polarization. However, the nature of the factors responsible for this regulation has remained primarily unknown. We have now addressed this issue and identified Lfc and Tctex-1 as two major interacting and opposing regulators of RhoA activity during axon formation.

Our data show that in young cultured hippocampal pyramidal neurons, Lfc promotes RhoA activity, thereby exerting a negative control that limits neurite sprouting, axon outgrowth, elongation, and branching. Several lines of evidence support this idea. First, Lfc levels modulate RhoA activity; Lfc overexpression in cultured neurons increases RhoA-GTP levels, whereas its suppression has the opposite effect. Confocal and TIRF microscopy shows that Lfc is prominent at the growth cone $\mathrm{P}$ domain, where high RhoA activity has been detected ( Na- 
Table 1. Effect of Lfc suppression on neuronal shape parameters

\begin{tabular}{llllll}
\hline Groups & $\begin{array}{l}\text { Number of } \\
\text { axons per cell }\end{array}$ & $\begin{array}{l}\text { Axonal length } \\
\text { per cell }\end{array}$ & $\begin{array}{l}\text { Number of } \\
\text { axonal branches }\end{array}$ & $\begin{array}{l}\text { Length of } \\
\text { minor processes }\end{array}$ & $\begin{array}{l}\text { Number of } \\
\text { minor processes }\end{array}$ \\
\hline SC-sh-HcRed & $1.36 \pm 0.11$ & $589 \pm 43$ & $11 \pm 1.8$ & $36 \pm 1.8$ & $4.96 \pm 0.42$ \\
sh1-LfC-HcRed & $2.40 \pm 0.18^{*}$ & $1070 \pm 48^{*}$ & $32 \pm 2.6^{*}$ & $42 \pm 1.3$ & $6.10 \pm 0.52$ \\
\hline
\end{tabular}

All measurements were performed at $3 \mathrm{~d}$ in vitro. Neurons were transfected at $2 \mathrm{~d}$ in vitro and fixed $24 \mathrm{~h}$ later. Two micrograms of plasmid were used for this experiment. Each value represents the mean $\pm \mathrm{SEM}$ of at least 30 cells for each experimental condition. Statistical analysis was determined by one-way ANOVA, followed by a Tukey's honestly significant difference post hoc test to compare SC-sh-HcRed- versus sh1-Lfc-HcRed-transfected neurons ( $\left.{ }^{*} p<0.0002\right)$.
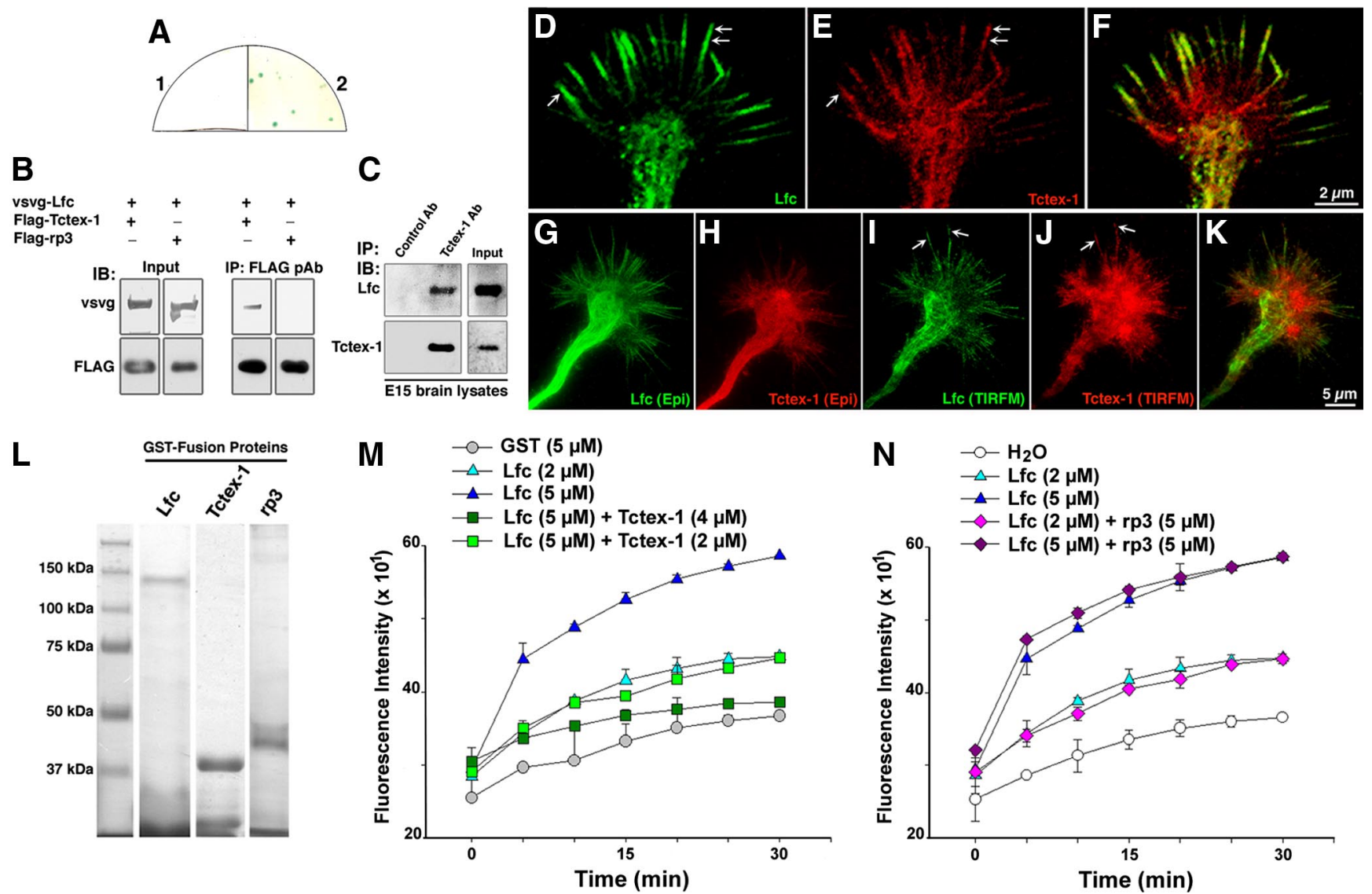

Figure 5. Tctex-1 interacts with Lfc and inhibits its GEF activity. A, Yeast two hybrid reveals an interaction between Tctex-1 and Lfc. 1, Negative control bait/prey, Lfc; 2, bait, Tctex-1/prey, Lfc. B, Immunoprecipitates of cell extracts from HEK cells transfected with vsvg-Lfc plus Flag-Tctex-1 or Flag-rp3. Note that Flag-Tctex-1, but not Flag-rp3, immunoprecipitated vsvg-Lfc. C, Control antibody (Control Ab) and Tctex-1 antibody (Tctex-1 Ab) immunoprecipitates performed on embryonic day 15 (E15) rat brain lysates and probed for Tctex-1 or Lfc; note the presence of Lfc in the Tctex-1 immunoprecipitates. D-F, A high-power confocal view of a large axonal growth cone from a stage 2-3 neuron showing the distribution of Lfc (green) and Tctex-1 (red). Note that both proteins colocalized within the growth cone (domain and at the distal end of filopodial extensions (arrows); Tctex-1 immunofluorescence was also present at the transition zone between the C and P domains, as well as at the base of filopodial extensions. G- $\boldsymbol{K}$, A series of images showing the distribution of $\mathrm{Lfc}$ (green) and Tctex-1 (red) in an axonal growth cone as revealed by epifluorescence and TIRFM. Note that TIRFM images also revealed colocalization of Lf and Tctex-1 within the growth cone P domain, including filopodial extensions (arrows). $L$, Coomassie blue staining of GST-fusion proteins used for the Lfc GEF assay. $M$, Graphs showing the GEF activity of GST-Lfc in the presence or absence of GST-Tctex-1. Note that GST-Lfc activity is inhibited in a dose-dependent manner by GST-Tctex-1; this inhibition was statistically significant $(p<0.001)$ after 5 min of initiating the assay. $\mathbf{N}$, A similar GEF assay showing the GST-rp3 had no effect on GST-Lfc activity. IP, Immunoprecipitates; IB, immunoblots; pAb, polyclonal antibody.

kamura et al., 2005). General targets of the RhoA-RhoK pathway such as TIAM1/STEF or LIMK1 are abundant at this region of the growth cone (Kunda et al., 2001; Rosso et al., 2004). In contrast, low RhoA activity has been reported in the growth cone $\mathrm{C}$ domain (Nakamura et al., 2005). Thus, the Lfc immunofluorescence signal associated with the $\mathrm{C}$ domain, where microtubules predominate, likely represents inactive Lfc (Birkenfeld et al., 2008). Second, Lfc overexpression phenocopies RhoA gain of function; Lfc reduces neurite sprouting and arrests axon outgrowth and elongation. This inhibitory effect is rescued by inhibiting RhoK. Third, silencing of Lfc results in a phenotype similar, if not identical, to that caused by overexpressing the Rho inhibitory toxin C3 (Da Silva et al., 2003) or treatment with Y27632 (Da Silva et al., 2003; Sanchez et al., 2008). Specifically, our results show that when Lfc is suppressed, neurons not only extend longer and highly branched axons but also form supernumerary ones. Together, our observations suggest a simple scenario in which all minor neurites have at least the minimal machinery required to activate axon growth but are prevented from doing so because of the existence of an Lfc-mediated RhoA-RhoK inhibitory tone. This signal appears to operate with equal intensity before and after polarization in those neurites not selected as axons. Accordingly, the selective or global downregulation of this inhibitory influence could be sufficient to trigger single or multiple axon formation, respectively.

Tctex-1 antagonizes $\mathrm{Lfc}$ activity to promote axonal growth The question that now arises concerns the factors that prevent the inhibitory function of Lfc-RhoA and their mechanism of action. 

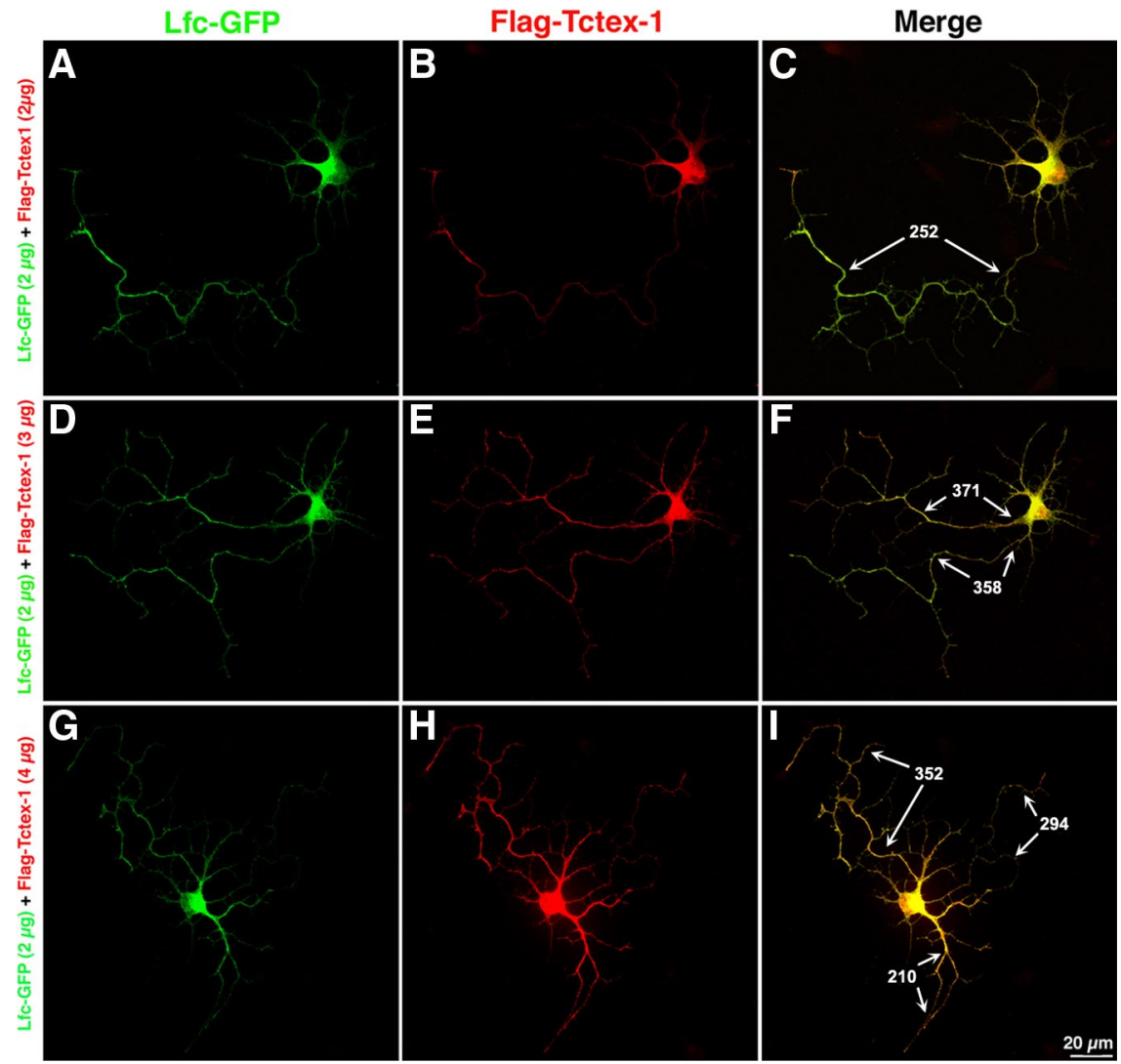

Figure 6. Tctex-1 counteracts the inhibitory effect of LfC on axon formation. A-I, Confocal images showing hippocampal pyramidal neurons after cotransfection of Lfc-GFP (green) and Flag-Tctex-1 (red). Neurons were transfected $4 \mathrm{~h}$ after plating and fixed $20 \mathrm{~h}$ later. Note that ectopic expression of Tctex-1 promotes axon formation, elongation, and branching in neurons coexpressing $L f($ and that this effect was dose dependent. The numbers in $\boldsymbol{C}, \boldsymbol{F}$, and $\boldsymbol{I}$ correspond to the length (micrometers) of axonal processes.

Inhibition of Lfc-RhoA may be achieved by either an increase in the activity of positive cues that counterbalance RhoA activity (e.g., enhanced expression of a Rac GEF such as Tiam1 or STEF) (Kunda et al., 2001), or by the action of proteins such as p190RhoGAP that interacts with aPKC-Par3/6 pathways and inhibits RhoA activity (Zhang and Macara, 2008) or SMURF1 (SMAD-specific E3 ubiquitin protein ligase 1), promoting RhoA ubiquitin/proteasome-mediated degradation (Bhupinder et al., 2007).

An alternative and effective way could be the direct targeting and inhibition of Lfc. The present results suggest that Tctex-1 could downregulate Lfc GEF activity and thereby antagonize its inhibitory action on axon formation. Several lines of evidence support this notion. First, Tctex-1 promotes neurite initiation, sprouting, and axon formation (Chuang et al., 2005), which is the opposite effect from that of Lfc-RhoA. This is consistent with a recent study showing that $L f c$ and Tctex- 1 have opposite actions in the regulation of cortical neurogenesis (Gauthier-Fisher et al., 2009). Second, Tctex-1 directly interacts with Lfc and inhibits its GEF activity in a dose-dependent manner. This inhibitory effect appears to be specific, since it is not exerted by rp3. In this regard, it is worth noting that Lfc overexpression is accompanied by a downregulation of Racl activity (Kang et al., 2009), whereas overexpression of Tctex-1 has the opposite effect (Chuang et al., 2005). Third, Lfc and Tctex-1 colocalize extensively within growth cones of developing neurites, including the large growth cone observed at the transition between stage 2 and 3 that marks an initial step in axon formation (Bradke and Dotti, 1997, 1999;
Kunda et al., 2001). Interestingly, Tctex-1, but not other dynein-associated proteins, becomes considerably enriched in the distal axonal shaft and its growth cone after reaching stage 3 (Chuang et al., 2005). Lfc (this study) and Tctex-1 (Tai et al., 1998) are also present in the Golgi apparatus, where other RhoA targets implicated in axon formation (e.g., LIMK1) have been identified (Rosso et al., 2004). Finally, the ectopic expression of Tctex-1 effectively antagonizes the inhibitory effect of Lfc overexpression on axon formation.

Our data coherently support a model in which Tctex-1 counterbalances the inhibitory influence of Lfc, thereby reducing RhoA-RhoK activity during axon formation. This antagonizing activity is likely to be much stronger in the axon, where Tctex- 1 accumulates considerably and may initiate the formation of a self-activating polarity module, including TIAM1, STEF, the Par polarity complex, Cdc42, and Racl that drives axon outgrowth and elongation. Additional factors may promote/enhance this activity. For example, DOCK7-mediated Rac activation leads to phosphorylation and inactivation of stathmin (Watabe-Uchida et al., 2006), a microtubule-destabilizing factor, contributing to the maintenance of a pool of microtubule-associated Lfc and thus reduced RhoA activity (Conde and Caceres, 2009). In the case of minor processes, the presence of Tctex-1 may be required to initiate sprouting (Chuang et al., 2005) and to prevent Lfc-RhoA mediated neurite retraction. However, this antagonizing influence is probably much weaker than in axons, allowing for a Rho inhibitory tone to prevail and prevent minor neurites from becoming axons.

\section{Final considerations}

While the above discussed observations highlight an inhibitory role for RhoA-RhoK in neurite sprouting and axogenesis, it should be mentioned that this pathway is also required for myosin II-driven microtubule bundling (Zhang et al., 2005; Burnette et al., 2008). This phenomenon, associated with actin arc contractility, occurs at the growth cone transition zone and is required for axon consolidation. Since we detected a low Lfc immunofluorescence signal in this growth cone region, it is possible that other factors regulate RhoA-RhoK activity at this location. It will now be of considerable interest to address the mechanisms underlying this regulation.

\section{References}

Aijaz S, D’Atri F, Citi S, Balda MS, Matter K (2005) Binding of GEF-H1 to the tight junction-associated adaptor cingulin results in inhibition of Rho signaling and G1/S phase transition. Dev Cell 8:777-786.

Arimura A, Kaibuchi K (2007) Neuronal polarity: from extracellular signals to intracellular mechanisms. Nat Rev Neurosci 8:194-205.

Barnes AP, Polleux F (2009) Establishment of axon-dendrite polarity in developing neurons. Annu Rev Neurosci 32:347-381.

Bhupinder P, Vohra S, Fu M, Heuckeroth RO (2007) Protein kinase Czeta and glycogen synthase kinase-3beta control neuronal polarity in developing rodent enteric neurons, whereas $\mathrm{SMAD}$-specific E3 ubiquitin protein 
ligase 1 promotes neurite growth but does not influence polarity. J Neurosci 27:9458-9468.

Birkenfeld J, Nalbant P, Yoon S, Bokoch G (2008) Cellular functions of GEF-H1, a microtubule regulated Rho-GEF: is altered GEF-H1 activity a crucial determinant of disease pathogenesis? Trends Cell Biol 18:210219.

Bisbal M, Conde C, Donoso M, Bollati F, Sesma J, Díaz Añel A, Quiroga S, Malhotra V, Marzolo M, Cáceres A (2008) Protein kinase D regulates trafficking of dendritic membrane proteins in developing neurons. J Neurosci 28:9297-9308.

Bisbal M, Wojnaki J, Peretti D, Roppolo A, Sesma J, Jausoro I, Cáceres A (2009) KIF4 mediated anterograde translocation and positioning of ribosomal constituents to developing axons. J Biol Chem 284:9489-9497.

Bito H, Furuyashiki T, Ishihara H, Shibasaki Y, Ohashi K, Mizuno K, Maekawa M, Ishizaki T, Narumiya S (2000) A critical role for a Rhoassociated kinase, p160ROCK, in determining axon outgrowth in mammalian CNS neurons. Neuron 26:431-441.

Borisoff JF, Carmen CM, Chan GW, Hiebert LO, Oschipok L, Robertson GS, Zamboni R, Steeves JD, Tetzlaffa W (2003) Suppression of Rho-kinase activity promotes axonal growth on inhibitory CNS substrates. Mol Cell Neurosci 22:405-416.

Bradke F, Dotti CG (1997) Vectorial cytoplasmic flow precedes axon formation. Neuron 19:1175-1186.

Bradke F, Dotti CG (1999) The role of local actin instability in axon formation. Science 283:1931-1934.

Burnette DT, Ji L, Schaefer AW, Medeiros NA, Danuser G, Forscher P (2008) Myosin II activity facilitates microtubule bundling in the neuronal growth cone neck. Dev Cell 2008 15:163-169.

Chan TO, Rodeck U, Chan AM, Kimmelman AC, Rittenhouse SE, Panayotou G, Tsichlis PN (2002) Small GTPases and tyrosine kinases coregulate a molecular switch in the phosphoinositide 3-kinase regulatory subunit. Cancer Cell 1:181-191.

Chuang J, Yen T, Bollati F, Conde C, Cáceres A, Sung C-H (2005) The dynein light chain Tctex-1 has a dynein-independent role in actin remodeling during neurite outgrowth. Dev Cell 9:75-86.

Chuang JZ, Milner TA, Sung CH (2001) Subunit heterogeneity of cytoplasmic dynein light chains in rat hippocampus. J Neurosci 21:5501-5512.

Conde C, Cáceres A (2009) Microtubule dynamics in axons, dendrites and at the synapse. Nat Rev Neurosci 10:319-332.

Da Silva JS, Medina M, Zuliani C, Di Nardo A, Witke W, Dotti CG (2003) RhoA/ROCK regulation of neuritogenesis via profilin Ila-mediated control of actin stability. J Cell Biol 162:1267-1279.

Gauthier-Fisher A, Lin DC, Graves M, Kaplan DE, Rottapel R, Miller F (2009) Lfc and Tctex-1 regulate the genesis of neurons from cortical precursor cells. Nat Neurosci 12:735-744.

Govek EE, Newey SE, Van Aelst L (2005) The role of the Rho GTPases in neuronal development. Genes Dev 19:1-49.

Kang M-G, Guo Y, Huganir RL (2009) AMPA receptor and GEF-H1/Lfc complex regulates dendritic spine development through RhoA signaling cascade. Proc Natl Acad Sci U S A 106:3549-3554.

Keely PJ, Westwick JK, Whitehead IP, Der CJ, Parise LV (1997) Cdc42 and Racl induce integrin-mediated cell motility and invasiveness through PI3K. Nature 390:632-636.

Kunda P, Paglini G, Kosik K, Quiroga S, Cáceres A (2001) Evidence for the involvement of Tiam-1 in axon formation. J Neurosci 21:2361-2372.
Meiri D, Greeve MA, Brunet A, Finan D, Wells CD, Larose J, Rottapel R (2009) Modulation of RhoGEF Lfc activity by PKA-mediated phosphorylation. Mol Cell Biol 29:5963-5973.

Nakamura T, Aoki K, Matsuda M (2005) FRET imaging in nerve growth cones reveals a high level of RhoA activity within the peripheral domain. Mol Brain Res 139:277-287.

Nakayama M, Goto TM, Sugimoto M, Nishimura T, Shinagawa T, Ohno S, Amano M, Kaibuchi K (2008) Rho-kinase phosphorylates Par3 and disrupts Par complex formation. Dev Cell 14:205-215.

Nishimura T, Yamaguchi T, Kato K, Yoshizawa M, Nabeshima Y, Ohno S, Hoshino M, Kaibuchi K (2005) PAR-6-PAR-3 mediates Cdc42 induced Rac activation through the Rac GEFs STEF/Tiam1. Nat Cell Biol $7: 270-277$.

Rosso S, Bollati F, Bisbal M, Peretti D, Sumi T, Nakamura T, Quiroga S, Ferreira A, Cáceres A (2004) LIMK1 regulates Golgi dynamics, traffic of Golgi-derived vesicles, and process extension in primary cultured neurons. Mol Biol Cell 15:3433-3449.

Ryan XP, Alldritt J, Svenningsson P, Allen PB, Wu GY, Nairn AC, Greengard $P$ (2005) The Rho-specific GEF Lfc interacts with neurabin and spinophilin to regulate dendritic spine morphology. Neuron 47:85-100.

Sanchez M, Gastaldi L, Remedi M, Cáceres A, Landa C (2008) Rotenoneinduced toxicity is mediated by Rho-GTPases in hippocampal neurons. Toxicol Sci 104:352-361.

Schwamborn JC, Puschel AW (2004) The sequential activity of the GTPases Rap1B and Cdc42 determines neuronal polarity. Nat Neurosci 7:923-929.

Shi SH, Jan L, Jan YN (2003) Hippocampal neuronal polarity specified by spatially localized mPar3/mPar6 and PI3-kinase activity. Cell 112:63-75.

Sordella R, Van Aelst L (2008) Dialogue between RhoA/ROCK and members of the Par complex in cell polarity. Dev Cell 14:150-152.

Sosa L, Dupraz S, Laurino L, Bollati F, Bisbal M, Cáceres A, Pfenninger K, Quiroga S (2006) IGF-1 receptor is essential for the establishment of hippocampal neuronal polarity. Nat Neurosci 9:993-995.

Tai AW, Chuang J-Z, Sung C-H (1998) Localization of Tctex-1, a cytoplasmic dynein light chain, to the Golgi apparatus and evidence for dynein complex heterogeneity. J Biol Chem 273:19639-19649.

Tai AW, Chuang JZ, Bide C, Wolfrum U, Sung CH (1999) Rhodopsin's carboxy-terminal tail acts as a membrane receptor for cytoplasmic dynein by binding to the dynein light chain Tctex-1. Cell 98:877-887.

Tashiro A, Yuste R (2008) Role of Rho GTPases in the morphogenesis and motility of dendritic spines. Methods Enzymol 439:285-302.

Tolias KF, Cantley LC, Carpenter CL (1995) Rho family GTPases bind to phosphoinositide kinases. J Biol Chem 270:17655-17659.

Vallee RB, Williams JC, Varma D, Barnhart LE (2004) Dynein: an ancient motor protein involved in multiple modes of transport. J Neurobiol 58:189-200.

Watabe-Uchida M, John KA, Janas JA, Newey SA, Van Aelst L (2006) The rac activator DOCK7 regulated neuronal polarity through local phosphorylation of stathmin/Op18. Neuron 51:727-739.

Zhang H, Macara I (2008) The Par-6 polarity protein regulates dendritic spine morphogenesis through p190RhoGAP and the RhoGTPase. Dev Cell 14:216-226.

Zhang XF, Schaefer AW, Burnette DT, Schoonderwoert V, Forscher P (2005) Rho-dependent contractile responses in the neuronal growth cone are independent of classical peripheral retrograde actin flow. Neuron 40:931944. 